\title{
MICROSTRUCTURAL CHANGES OF THE CORPUS CALLOSUM BY MAGNETIC RESONANCE IMAGING IN ADULT PATIENTS WITH SYSTEMIC LUPUS ERYTHEMATOSUS ACCORDING TO THE AGE OF DISEASE ONSET
}

Paulo Rogério Julio ${ }^{1}$, Carla Helena Cappello ${ }^{1}$, Simone Thiemi Kishimoto ${ }^{1}$, Renan Bazuco Frittoli ${ }^{1}$, Roberto Marini ${ }^{1}$, Fernando Cendes $^{1}$, Leticia Rittner ${ }^{1}$, Lilian Tereza Lavras Costallat ${ }^{1}$, Simone Appenzeller ${ }^{1, *}$

1.Universidade Estadual de Campinas, Campinas (SP), Brazil.

*Corresponding author: appenzellersimone@gmail.com

\section{BACKGROUND}

Neuropsychiatric (NP) manifestations in childhood-onset systemic lupus erythematosus (cSLE) has been shown to be more severe and associated with greater burden than in adult-onset (aSLE). However, the presence of structural abnormalities seen on magnetic resonance imaging (MRI) cannot solely explain these findings. Microstructural changes using diffusion tensor imaging (DTI) is used to evaluate the microstructure of the white matter. The aim of this study was to determine with the age of onset of disease could influence the presence of corpus callosum (CC) atrophy.

\section{MATERIALS AND METHODS}

Seventy-one patients with cSLE [mean age of 24.7 years old (SD 4.6) and disease duration of 11.8 years (SD 4.8)], 49 patients with aSLE [mean age 33.2 (SD 3.7)] and disease duration 11.3 years (SD 4.05)] and 58 healthy controls (HC) [mean age of 29.9 years old (SD 4.1)] were included. Three-tesla magnetic resonance imaging (3T-MRI) was obtained and all individuals had a complete neurological and psychiatric evaluation performed on the day of the MRI.

\section{RESULTS}

We did not observe significant differences between the volumes of CC in the three groups evaluated. In the assessment of total DTI, we observed significantly reduction of FA [0.63603; $(p \quad 0.001)$ SD $\pm 0.0665 ; p=0.002$ ], and increased values of MD (0.00096; $\mathrm{SD} \pm 0.00014 ; p=0.002), \mathrm{RD}(0.00057 ; \mathrm{SD} \pm 0.00015 ; p=0.002)$ and $\mathrm{AD}(0.0017 ; \mathrm{SD} \pm 0.00014 ; p=0.024)$ in cSLE when compared with aSLE. Decreased FA values and increased MD values in the group of patients with cSLE were associated with the presence of NP manifestations when compared to the group of patients with aSLE.

\section{CONCLUSION}

Microstructural abnormalities, despite normal structural MRI findings can explain the greater severity and burden associated with NP involvement in cSLE. 